\title{
Quantized Conductance and Its Correlation to the Supercurrent in a Nanowire Connected to Superconductors
}

\author{
Simon Abay, ${ }^{* \dagger}$ Daniel Persson, $^{\dagger}$ Henrik Nilsson, $^{\ddagger}$ H. Q. Xu, ${ }^{\ddagger}$, Mikael Fogelström, $^{\dagger}$ Vitaly Shumeiko, $^{\dagger}$ \\ and Per Delsing*, $\dagger$
${ }^{\dagger}$ Department of Microtechnology and Nanoscience (MC2), Chalmers University of Technology, SE-412 96 Göteborg, Sweden
${ }^{\ddagger}$ Division of Solid State Physics, Lund University, Box 118, S-221 00 Lund, Sweden
${ }^{\S}$ Key Laboratory for the Physics and Chemistry of Nanodevices and Department of Electronics, Peking University, Beijing 100871, China

ABSTRACT: We report conductance and supercurrent of InAs nanowires coupled to Al-superconducting electrodes with short channel lengths and good Ohmic contacts. The nanowires are suspended $15 \mathrm{~nm}$ above a local gate electrode. The charge density in the nanowires can be controlled by a small change in the gate voltage. For large negative gate voltages, the number of conducting channels is reduced gradually, and we observe a stepwise decrease of both conductance and critical current before the conductance vanishes completely.
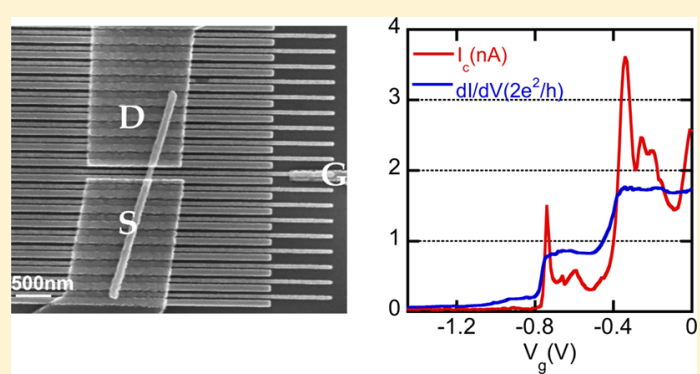

KEYWORDS: InAs nanowires, supercurrent, Andreev reflection, quantum of conductance

$\mathrm{T}^{\mathrm{k}}$ he ability to control physical properties and chemical composition of semiconducting nanowires has made them attractive devices to study quantum effects at a very small scale. In particular, semiconducting nanowires coupled to superconductors have been attractive to study quantum transport effects such as quantum interference, ${ }^{1}$ Cooper-pair field effect, ${ }^{2,3}$ and Cooper-pair beam splitting. ${ }^{4}$ More recently, they have been used to confirm the presence of exotic states in condensed matter, Majorana Fermion bound states. ${ }^{5-8}$

Semiconducting weak links offer the possibility to gatecontrol the number of conducting channels, and hence, allow to tune the coupling strength of the weak links. For a ballistic onedimensional weak link, the conductance is expected to increase in quantized steps as a function of gate voltage. ${ }^{9}$ In point contacts, defined in two-dimensional electron gas (2DEG) heterostructure devices by a split-gate, charge transport can be ballistic, and the conductance can be quantized. Evidences of conductance quantization have been realized by many groups in such gate-controlled point contacts. ${ }^{10,11}$ However, quantization of conductance was only recently reported in nanowires at nonzero magnetic field. ${ }^{12}$ In nanowire weak links this has been difficult mainly due to the scattering of electrons in the conduction paths: reflections of electrons due to scattering centers such as crystal defects, impurities, Schottky barriers, and surface states. Backscattering by such inhomogeneities smears out the conductance steps. In a weak link that is connected to superconducting electrodes, there will also be a supercurrent, and, in line with the conductance quantization, the critical current through such a weak link is also predicted to be quantized with a step height of $\delta I_{c}=e \Delta / \hbar{ }^{13}$ Critical current steps has been previously reported in 2DEG point contacts $^{14-16}$ but the observed current steps have been substantially smaller than those predicted by theory. In this paper, we report quantization of conductance in suspended InAs nanowire devices with short channel lengths and good Ohmic contact interfaces. We also observe steps in the critical current that are correlated with the onset of the conductance steps.

The geometry of the suspended devices offers the possibility to easily gate-control the number of conducting channels in the nanowires. The control of a few number of conducting channels with nearly ballistic transport will be an important contribution to the ongoing efforts to create topological states, such as Majorana states. ${ }^{5-8}$

The nanowires are grown by chemical beam epitaxy. ${ }^{17}$ They have an average diameter of $80 \mathrm{~nm}$ and an average length of 4 $\mu \mathrm{m}$. To fabricate the suspended devices, a standard Si-substrate capped by $400 \mathrm{~nm}$ thick $\mathrm{SiO}_{2}$ is first patterned with interdigitated $\mathrm{Ti} / \mathrm{Au}$ stripes. ${ }^{18}$ The InAs nanowires are then transferred to the already patterned $\mathrm{Si}$ substrate, and some of the nanowires end up on top of the interdigitated metal stripes. The interdigitated metal stripes are made in a two-step nanofabrication process in order to get a height difference of $15 \mathrm{~nm}$ between every two adjacent stripes. This allows the nanowire to rest on the thicker electrodes while being suspended above the substrate and the thinner electrodes. With the help of scanning electron microscope (SEM) images, the positions of suitable nanowires are found and super-

Received: April 20, 2013

Revised: July 15, 2013

Published: July 30, 2013 
conducting electrodes $(\mathrm{Ti} / \mathrm{Al}(5 / 150) \mathrm{nm}$ thick) are defined on selected nanowires with electron beam lithography. A SEM image of a suspended device is shown in Figure 1a. To get good

a)
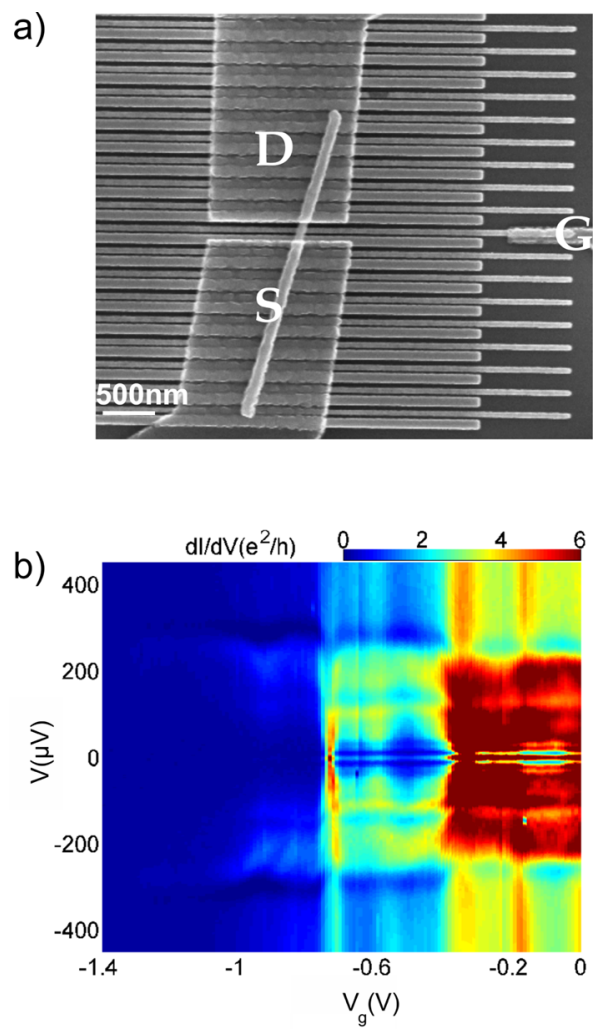

Figure 1. (a) A SEM image of a suspended device. The interdigitated $\mathrm{Ti} / \mathrm{Au}$ stripes are fabricated with a thickness difference of $15 \mathrm{~nm}$ such that the nanowire is suspended above the local gate and the substrate. (b) An image plot of a differential conductance $\mathrm{d} I / \mathrm{d} V$ as a function of gate and source-drain voltages. The differential conductance is recorded at the base temperature of $15 \mathrm{mK}$. We observe two conductance changes along the gate voltage which mark the opening of the first and the second channels. Similarly, once the first channel starts to conduct, the conductance changes with the source-drain voltage as the transport crosses over from Andreev transport to quasiparticle transport at $V=2 \Delta / e \approx 250 \mu \mathrm{V}$.

transparency of the interfaces, an ammonium polysulfide solution $\left(\mathrm{NH}_{4} S_{x}\right)$ cleaning process ${ }^{19,20}$ was used prior to evaporation of the superconducting contacts. The samples are then characterized at room temperature and stored in vacuum before further measurements at low temperatures.

Current-voltage characteristics (IVCs) of the devices were measured in a dilution refrigerator with a base temperature of $15 \mathrm{mK}$. To decrease noise coupling to the devices, the electrical lines in the measurement set up are well filtered and thermally anchored at different temperature stages of the refrigerator.

A typical device with a nearby local gate is shown in Figure 1a. We have measured several suspended devices with a broad range of lengths $L$ and normal state resistances $R_{\mathrm{n}}$. In general, the devices exhibited intrinsic n-type behavior such that the overall normal state resistance decreased as the gate voltage was increased from negative to positive voltages. The differential conductance $\mathrm{d} I / \mathrm{d} V$ as a function of gate voltage $V_{\mathrm{g}}$ and sourcedrain voltage $V$ is shown in Figure $1 \mathrm{~b}$ for a junction with $L \approx$ $150 \mathrm{~nm}$ and $R_{\mathrm{n}}=6.7 \mathrm{k} \Omega$ at $V_{\mathrm{g}}=0 \mathrm{~V}$. We observe transition from tunneling regime to conducting regime and stepwise increases of the conductance due to the opening of conducting channels. These conductance changes mark the opening of the first and the second channels with increasing gate voltage. Similarly, along the vertical axis, in the conducting regime, we observe a major conductance change at the voltage of $|2 \Delta / e| \sim$ $250 \mu \mathrm{V}$, where the transport crosses over from Andreev transport at low bias to single-particle transport at high bias.

To clearly display the observed conductance changes as a function of the gate voltage, two line cuts of the differential conductance are shown in Figure 2a. The line cuts correspond
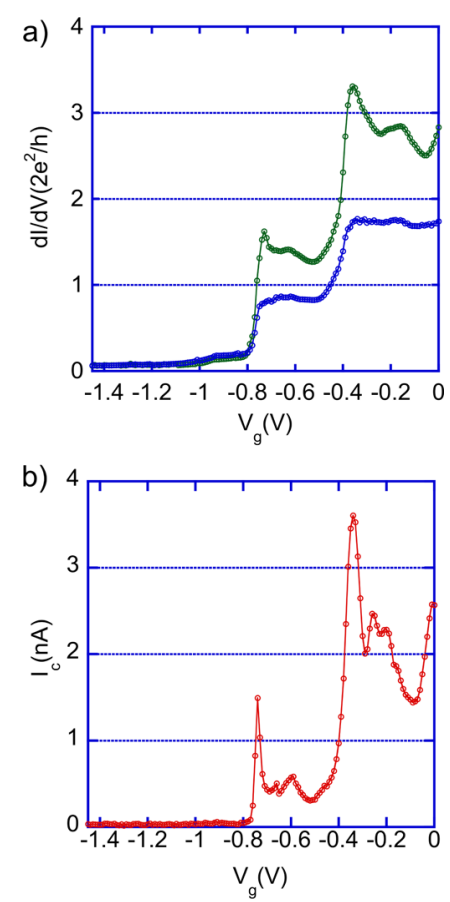

Figure 2. (a) Normal state conductance (blue) and a subgap conductance (green) as a function of gate voltage. The normal state conductance, extracted at $V=1 \mathrm{mV}$, clearly displays a stepwise increase of the conductance as a function of the gate voltage. The subgap conductance, taken at $V=125 \mu \mathrm{V} \sim \Delta / e$, also increases in steps but with step height larger than the normal state conductance. (b) The critical current as a function of the gate voltage. The critical current also increases in steps directly correlated with the normal state conductance.

to two source-drain voltages, at large bias, $V=1 \mathrm{mV} \gg 2 \Delta$ /e (blue), where the conductance corresponds to the normal state value, and the subgap state $V=125 \mu \mathrm{V} \sim \Delta / e$ (green). The normal state differential conductance $\mathrm{d} I / \mathrm{d} V$ at $V \gg 2 \Delta$ /e displays clear quantization of the conductance as a function of the gate voltage. As the gate voltage is increased to more positive values, the potential barrier height decreases, and the channels become conducting one by one. The measured step heights are almost equal to one quantum of conductance $2 e^{2} / h$ owing to the short channel lengths and indicating almost perfect transmission of the conducting channels. The intrinsic interface resistance due to the Fermi-velocity mismatch and the nonzero resistance of the Ohmic contacts explain the small deviations in the step height from the ideal quantum of conductance.

The subgap conductance also increases stepwise as a function of the gate voltage. A differential conductance at a source-drain voltage of $V=125 \mu \mathrm{V} \sim \Delta / e$ (green) is shown in Figure 2a. The measured step height is bigger than one quantum of 
conductance $\left(2 e^{2} / h\right)$, which is attributed to the two-particle charge transport mechanism of Andreev reflection. ${ }^{21,22}$

Next, we present the critical current as a function of the gate voltage, as shown in Figure 2b. At high negative gate voltages, the critical current is completely suppressed in the same way as the normal state conductance. When the gate voltage reaches $V_{\mathrm{g}} \approx-0.8 \mathrm{~V}$, at which the first channel starts to conduct, the critical current starts to appear. It shows a peak of $1.5 \mathrm{nA}$ at the channel onset, but then rapidly decreases to a plateau with an average of $0.5 \mathrm{nA}$. When the gate voltage was further increased to open a second conducting channel, the critical current again shows a peak (to $3.6 \mathrm{nA}$ ), a resonant structure, and then settles down to an average of $2 \mathrm{nA}$. Such resonant structures in the supercurrent were suggested by Furusaki et al. ${ }^{24}$ and attributed to interference between electrons and holes being reflected at a constriction. The peaks we observe show a qualitative agreement with such a scenario. The stepwise increase of the critical current is directly correlated to the stepwise increase of the normal state conductance. However, the observed step height is substantially lower than the maximum theoretical value $I_{c}(T)=e \Delta / \hbar\left(1-(1-T)^{1 / 2}\right) \approx 14 \mathrm{nA}^{25}$ where the transmission probability of the first channel $T \approx 0.8$ is estimated from the step height of the normal state conductance. Suppression of the critical current has been observed in Josephson junction devices embedding a weak link, ${ }^{14-16}$ including those made from a nanowire, ${ }^{2,3}$ and can at least partly be attributed to premature switching due to thermal activation or quantum tunneling of the phase. ${ }^{26}$

To observe the conductance quantization and the correlated critical current steps, high-quality interfaces are necessary. Here, we extract an additional information about the contact interface transparency by quantifying the excess current. ${ }^{21}$ We have plotted the IVC of the device in Figure 3a. The IVC is taken at $V_{\mathrm{g}}=0 \mathrm{~V}$, which corresponds to two open conduction channels. At high voltages, $V>|2 \Delta / e| \sim 250 \mu \mathrm{V}$, the IVC displays a linear behavior with a slope of the normal state conductance. Once the voltage reaches $V \sim 2 \Delta / e$, we observe enhancement of the conductance evident by the change of slope in the IVC. A linear fit (blue dashed line) to the normal state (above $2 \Delta$ ) does not extrapolate to the origin but to an excess current, $I_{\mathrm{exc}} \approx 23 \mathrm{nA}$. The interface transparency $T$ can be extracted from the extrapolated excess current. Using the formula for the excess current derived in ref 23 , we calculated the contact average transparency $T=0.67$, for $\Delta \sim 125 \mu \mathrm{eV}$, and $R_{\mathrm{n}}=6.7 \mathrm{k} \Omega$. The extracted value is of the same order as the one for the first step, and it is close to the transmission probability extracted from the normal resistance.

In order to clearly display the enhanced subgap conductance and the subgap structures, we have presented normalized differential resistance $\mathrm{d} V / \mathrm{d} I$ as a function of source-drain voltage for $V_{\mathrm{g}}=0 \mathrm{~V}$. As can be seen in Figure $3 \mathrm{~b}$, the resistance drops substantially when the device goes from quasi-particle transport to Andreev transport. The normal state resistance at $V \gg \mid 2 \Delta / e l$, is almost twice the subgap resistance at $V \ll|2 \Delta / e|$ (cf. experimental data for InAs 2DEG in ref 16, Figure 2, and theoretical calculation in ref 27 Figure 3 ). The plot also clearly presents anomalous multiple Andreev reflection (MAR) features at voltage positions corresponding to $(2 \Delta / n e)$. The first five, $n=1,2,3,4,5$ indicated by arrows in Figure $3 \mathrm{~b}$, scales with the superconducting gap. These results are similar to the differential resistance $\mathrm{d} V / \mathrm{d} I$ of quasi-ballistic InAs (2DEG) devices. ${ }^{16}$ However, the pronounced peak, near-zero bias
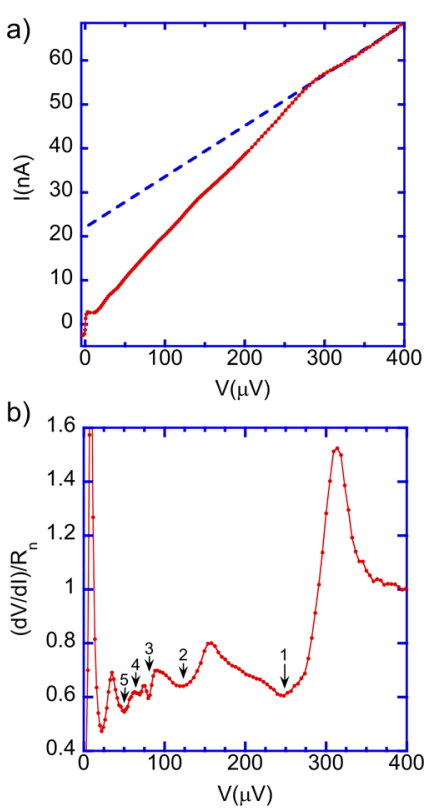

Figure 3. (a) Current voltage characteristic taken at $V_{\mathrm{g}}=0 \mathrm{~V}$ and at a temperature of $15 \mathrm{mK}$. Above $V>2 \Delta / e$, the current voltage characteristic shows a linear behavior with a normal state resistance of $R_{\mathrm{n}}=6.7 \mathrm{k} \Omega$. Once $V \leq 2 \Delta / e$, the current voltage characteristic shows an enhanced conductance evident by a change of slope (visible as a kink). The excess current is shown with a blue dashed line, a linear fit of the data in the normal state extrapolated to $V=0$. (b) Normalized differential resistance $(\mathrm{d} V / \mathrm{d} I) R_{n}$ of the current-voltage characteristics in panel a. The subgap resistance changes to almost half of the normal state resistance. Subgap features from multiple Andreev reflections are observed at $2 \Delta / n e$, where $n=1,2,3,4$, and 5 .

voltage does not depend on the superconducting gap, and we therefore believe that its origin is different from MAR.

In conclusion, we have fabricated devices of InAs nanowires suspended above a local gate electrode with short channel lengths and good Ohmic contacts. The local charge concentration and hence the conductance of the nanowires was quite sensitive to small changes of the gate voltage. As a function of the gate voltage, the normal state conductance increased in steps with step heights almost one conductance quantum $2 e^{2} / h$. Similarly, the subgap conductance and the critical current also increased in steps directly correlated with the normal state conductance.

\section{AUTHOR INFORMATION}

\section{Corresponding Author}

*E-mail: abay@chalmers.se (S.A.); per.delsing@chalmers.se (P.D.).

\section{Notes}

The authors declare no competing financial interest.

\section{ACKNOWLEDGMENTS}

We acknowledge fruitful discussions with Lars Samuelson, Christopher Wilson, and Jonas Bylander. The work was supported by the Swedish Research council, the Wallenberg foundation, and the National Basic Research Program of the Ministry of Science and Technology of China (Nos. 2012CB932703 and 2012CB932700). 


\section{REFERENCES}

(1) Doh, Y.; J., De Franceschi, S.; Bakkers, E. P. A. M.; Kouwenhoven, L. P. Nano Lett. 2008, 8, 4098.

(2) Doh, Y.; van Dam, J.; Roest, A.; Bakkers, E.; Kouwenhoven, L.; De Franceschi, S. Science 2005, 309, 272.

(3) Nilsson, H. A.; Samuelsson, P.; Caroff, P.; Xu, H. Q. Nano Lett. 2012, 12, 228.

(4) Hofstetter, L.; Csonka, S.; Nygard, J.; Schoenenberger, C. Nature 2009, 461, 960.

(5) Mourik, V.; Zuo, K.; Frolov, S. M.; Plissard, S. R.; Bakkers, E. P. A. M.; Kouwenhoven, L. P. Science 2012, 336, 1003.

(6) Deng, M. T.; Yu, C. L.; Huang, G. Y.; Larsson, M.; Caroff, P.; Xu, H. Q. Nano Lett. 2012, 12, 6414.

(7) Das, A.; Ronen, Y.; Most, Y.; Oreg, Y.; Heiblum, M.; Shtrikman, H. Nat. Phys. 2012, 8, 887.

(8) Churchill, H. O. H.; Fatemi, V.; Grove-Rasmussen, K.; Deng, M. T.; Caroff, P.; Xu, H. Q.; Marcus, C. M. Phys. Rev. B 2013, 87, 241401(R).

(9) Buttiker, M.; Imry, Y.; Landauer, R.; Pinhas, S. Phys. Rev. B 1985, 31, 6207.

(10) Van Wees, B. J.; et al. Phys. Rev. Lett. 1988, 60, 848.

(11) Wharam, D. A.; et al. J. Phys. C 1988, 21, L209.

(12) Ilse van Weperen; Plissard, S. R.; Bakkers, E. P. A. M.; Frolov, S. M.; Kouwenhoven, L. P. Nano Lett. 2013, 13, 387.

(13) Beenakker, C.; Van Houten, H. Phys. Rev. Lett. 1991, 66, 3056.

(14) Bauch, T.; Hürfeld, E.; Krasnov, V. M.; Delsing, P. Phys. Rev. B 2005, 71, 174502.

(15) Takayanagi, H.; Akazaki, T.; Nitta, J. Phys. Rev. Lett. 1995, 75, 3533.

(16) Samuelsson, P.; Ingerman, A.; Johansson, G.; Bezuglyi, E. V.; Shumeiko, V.; Wendin, G.; Kursten, R.; Richter, A.; Matsuyama, T.; Merkt, U. Phys. Rev. B 2004, 70, 212505.

(17) Ohlsson, B. J.; Björk, M. T.; Persson, A. I.; Thelander, C.; Wallenberg, L. R.; Magnusson, M. H.; Deppert, K.; Samuelson, L. Physica E. 2002, 13, 1126.

(18) Nilsson, H. A.; Duty, T.; Abay, S.; Wilson, C. M.; Wagner, J. B.; Thelander, C.; Delsing, P.; Samuelson, L. Nano Lett. 2008, 8, 872.

(19) Suyatin, D. B.; Thelander, C.; Björk, M. T.; Maximov, I.; Samuelson, L. Nanotechnology 2007, 18, 105307.

(20) Abay, S.; Nilsson, H. A.; Fan Wu, Xu, H.Q.; Wilson, C. M.; Delsing, P. Nano Lett. 2012, 12, 5622.

(21) Blonder, G. E.; Tinkham, M.; Klapwijk, T. M. Phys. Rev. B 1982, 25,4515 .

(22) Andreev, A. F. Zh. Eksp. Theor. Fiz. 1964, 46, 1823.

(23) Shumeiko, V. S.; Bratus, E. N.; Wendin, G. Low Temp. Phys. 1997, 23, 181.

(24) Furusaki, A.; Takayanagi, H.; Tsukada, M. Phys. Rev. B 1992, 45, 10563.

(25) Chtchelkatchev, N. M.; Lesovik, G. B.; Blatter, G. Phys. Rev. B 2000, 62, 3559.

(26) Tinkham, M. Introduction to Superconductivity; Dover: New York, 1996.

(27) Cuevas, C.; Hammer, J.; Kopu, J.; Viljas, J. K.; Eschrig, M. Phys. Rev. $B$ 2006, 73, 184505. 